\title{
Constitutive modeling of two phase materials using the mean field method for homogenization
}

\author{
E. Semih Perdahcıoğlu • Hubert J. M. Geijselaers
}

Received: 3 June 2010 / Accepted: 2 November 2010 / Published online: 25 November 2010

(C) The Author(s) 2010. This article is published with open access at Springerlink.com

\begin{abstract}
A Mean-Field homogenization framework for constitutive modeling of materials involving two distinct elastic-plastic phases is presented. With this approach it is possible to compute the macroscopic mechanical behavior of this type of materials based on the constitutive models of the constituent phases. Different homogenization schemes that exist in the literature are implemented in efficient algorithms to be used in fullscale FE simulations. These schemes are compared with each other in terms of efficiency. Additionally two new schemes are proposed which are both computationally efficient and compare in accuracy with the more physically based approaches. Finally the algorithms are demonstrated on FE simulations of sheet metal forming operations and compared with experimental results.
\end{abstract}

Keywords Mean field • Homogenization • Mori-Tanaka • Self consistent • Dual-phase steels • Metal-Matrix composites

E. S. Perdahcioğlu $(\bowtie)$

Materials innovation institute,

Faculty of Engineering Technology, Section of Applied

Mechanics, University of Twente, P.O. Box 217,

NL 7500AE Enschede, The Netherlands

e-mail: e.s.perdahcioglu@ctw.utwente.nl

H. J. M. Geijselaers

Faculty of Engineering Technology, Section of Applied

Mechanics, University of Twente, P.O. Box 217,

NL 7500AE Enschede, The Netherlands

\section{Introduction}

The growing needs for improved properties of products in terms of energy consumption, reduced weight, functionality, smaller dimensions etc. leads to the investigation of advanced materials that provide more with less. For the automotive industry for instance different grades of steels such as TRIP (TRansformation Induced Plasticity) and DP (Dual-Phase) steels are of interest. In personal care applications metastable austenitic stainless steels are being used for their unconventional mechanical and physical properties.

One common feature among these alloys is that they involve multiple phases in their microstructure. A family from these materials can even be tailored to yield different phase fractions with slight changes in the production routes. In this sense these materials can be considered as composite materials and since the main concern of this study is metals, it is justifiable to refer to them as Metal Matrix Composites (MMC). Clearly different approaches can be followed for modeling these materials. The conventional hardening models for instance can be used to fit their stressstrain curves. The drawback of this method is that for different batches with different phase fractions new mechanical tests have to be carried out for fitting. Additionally it is hard to use these models for tailoring new batches to suit a specific purpose. A physically based approach is presented here in which the most important factor influencing the macroscopic mechanical behavior is considered to be the mixture of two phases with different mechanical properties. Although each phase can be modeled with simplified phenomenological approaches, the mixture can possess totally different properties than each phase separately. 
In the current approach, the mechanical properties of the composite are approximated using the MeanField homogenization method. This method makes it possible to work with the averages of the fields within subdomains to compute the overall response. Previous studies show that this homogenization technique can be used successfully to compute mechanical response of reinforced solids (see for instance $[2,5]$ ).

The outline of this paper is as follows. First, the general ingredients of the homogenization technique are introduced. Different homogenization schemes as well as the solution algorithms developed are described next. Finally the results of the study are demonstrated on a sheet-metal forming simulation.

\section{Mean-field homogenization}

Homogenization is a general term that refers to the procedure of homogenizing the properties of an inhomogeneous material to obtain a higher level approximation. The main ingredients of this procedure are apparently averaging the fields over the volume of a domain and projecting the results on its boundary.

Homogenization techniques are generally based on the concept of a representative volume element (RVE) which was introduced by HIll [6]. An RVE represents a macroscopic point in the material (i.e. an integration point in a Finite Element model) as a finite sized inhomogeneous volume that is representative for the microstructure of the material. The aim therefore is to solve a complementary problem on the RVE and then use the results in macroscale.

One of the possibilities of using the RVE for homogenization is to solve a finite element model defined over the RVE using as the boundary conditions a combination of the macroscopic values (see for instance $[9,10]$ ). The computed results are returned to the macroscale by suitable averaging techniques. This procedure is very accurate since a detailed analysis of the stresses and strains that develop in the RVE is done. On the other hand, it is clear that this method is computationally very expensive.

The Mean-Field method is built on the concept of an imaginary RVE rather than an actual one. The inhomogeneities are treated as separate homogeneous domains in an aggregate of domains. The fields over the subdomains are represented by their averaged values. The complementary problem then is to solve for the interaction of the subdomains and find the new averages that satisfy the boundary conditions. This is usually carried out by using analytical solutions to the simplified versions of the problem [1].
One of the main steps in homogenization is the scale transition which requires macroscopic quantities to be transferred to the lower scale and vice versa. This process is carried out using an averaging operator. The assumption therefore is that macroscopic quantities can be described with the averages of the microscopic fields over a volume. The averaging operator is defined as:

$\langle g(\mathbf{x})\rangle_{\omega}=\frac{1}{V} \int_{\omega} g(\mathbf{x}) \mathrm{d} V=\bar{g}$

where $g$ is any field defined over the domain, $\bar{g}$ is the macroscopic value and $\langle g(\mathbf{x})\rangle_{\omega}$ is the averaged value of $g$ over the coordinates $\mathbf{x}$ belonging to the volume $\omega$.

Equation 1 implies that the volume over which a field is averaged can be decomposed into different domains:

$\langle g(\mathbf{x})\rangle_{\omega}=\frac{1}{V} \sum_{k} \int_{\omega_{k}} g(\mathbf{x}) \mathrm{d} V_{k}, \quad \omega=\bigcup_{k} \omega_{k}$.

Rephrasing Eq. 2 in terms of volume fractions of the decomposed domains gives:

$\langle g(\mathbf{x})\rangle_{\omega}=\sum_{k} f_{k}\langle g(\mathbf{x})\rangle_{\omega_{k}}, \quad f_{k}=\frac{V_{k}}{V}$.

To be able to justify the scale transition for stress and strain, the consistency of the mechanical work computed in both scales must be assured. This requires that the averaged mechanical work over the RVE is equal to the mechanical work that is calculated using the averaged stress and strain. In mathematical terms, the condition requires:

$\overline{\boldsymbol{\sigma}}: \bar{\varepsilon}=\langle\boldsymbol{\sigma}\rangle_{\omega}:\langle\boldsymbol{\varepsilon}\rangle_{\omega}=\langle\boldsymbol{\sigma}: \boldsymbol{\varepsilon}\rangle_{\omega}$.

Equation 4 is known as the Hill-Mandel condition. According to Hill [6], a necessary condition for the validity of this condition is that equilibrium and compatibility within the RVE are satisfied. Furthermore, the boundary conditions applied on the surface of the RVE must be of the type that would produce uniform strain or stress within the RVE if the material were homogeneous.

Supported by the Hill-Mandel condition, scale transition relations for stress and strain are given as:

$\overline{\boldsymbol{\sigma}}=\sum_{k} f_{k}\langle\boldsymbol{\sigma}\rangle_{\omega_{k}}, \quad \overline{\boldsymbol{\varepsilon}}=\sum_{k} f_{k}\langle\boldsymbol{\varepsilon}\rangle_{\omega_{k}}$.

These equations are exact under the small strain assumption. In the case of finite strains and deformations, some considerations must be taken into account. One of the difficulties when dealing with large deformations is the volume over which the averaging operation is carried out. In finite deformations and strains, the reference and current configuration are different from each 
other. Some strain and stress measures are defined in the former and some in the latter. This causes an inconsistency in relating the macroscopic averages to the averaged values over the RVE. An excellent analysis for large deformation averaging procedures is given in Nemat and Nasser [14].

The equations presented so far only provide the scale transition relations once the fields are determined on the RVE. Using a Direct FE approach the fields can be computed by applying the macroscopic stress or strain in terms of surface boundary conditions (tractions and displacements, respectively) on the RVE. After the fields are computed numerically, averaging is carried out and the results are transferred to the macroscale. In the Mean-Field homogenization schemes, instead of the fields, only the averages in different sub-domains are computed based on a number of assumptions which will be described in the following section.

In order to compute the stress and strain fields on an RVE, the mechanical response of the phases must be known. Generally in the Mean-Field methods it is assumed that each constituent phase follows its own macroscopic material behavior. Furthermore, the effects of the interactions between the phases and their boundaries are neglected. With these assumptions, the relation between the average stress and strain on the sub-domains on the RVE is supplied by continuum material models:

$\langle\boldsymbol{\sigma}\rangle_{\omega_{k}}=\mathbb{C}_{k}:\langle\boldsymbol{\varepsilon}\rangle_{\omega_{k}}$

where $\mathbb{C}$ is the elasticity tensor.

Equation 6 provides the stress-strain relation for each phase individually. However, in order to compute either the stress or the strain, one of these quantities must be supplied. Therefore the equations given so far are not sufficient to solve the homogenization problem. The remaining equations must supply the relation between the strains or stresses among the different phases. These equations can be written in terms of strain or stress concentration relations:

$\langle\boldsymbol{\varepsilon}\rangle_{\omega_{k}}=\mathbb{A}_{k}:\langle\boldsymbol{\varepsilon}\rangle_{\omega}, \quad\langle\boldsymbol{\sigma}\rangle_{\omega_{k}}=\mathbb{B}_{k}:\langle\boldsymbol{\sigma}\rangle_{\omega}$

where $\mathbb{A}$ and $\mathbb{B}$ are the fourth-order strain and the stress concentration tensors, respectively. In the case when all the concentration tensors of one type are known, the tensors for the other type can be determined. MeanField homogenization schemes differ from each other by the selection of these tensors. In the following sections the schemes that are studied are discussed.

\section{Homogenization schemes}

The simplest schemes are Voigt and Reuss estimates where isostrain and isostress conditions among the phases are assumed, respectively. Hence in the Voigt scheme all the strain concentration tensors are equal to unity, i.e. $\mathbb{A}_{k}=\mathbb{I}$. This scheme gives an upper bound for the stress response of the composite. Similarly in the Reuss scheme the stress concentration tensors are unity, i.e. $\mathbb{B}_{k}=\mathbb{I}$ and it gives a lower bound for the stress response.

A better approach for determining the strain concentration tensor is to use Eshelby's analytical solution to the inhomogeneity problem. Eshelby [4] introduced the equivalent inclusion theory in order to solve the strain concentration problem for a single inhomogeneity embedded in an infinitely large matrix. Hence his solution can be used only for a composite with two phases: the matrix and the inhomogeneity. He showed that when the matrix properties are isotropic and the inhomogeneity has an ellipsoidal shape, the localized stress and strain fields in the inclusion are uniform.

\section{Eshelby's solution}

The problem is to find the strain concentration due to a single inhomogeneity in an infinitely large matrix. To arrive at the solution Eshelby first derives the inclusion theory. In this problem the equilibrium strain and stress are to be determined in the case where a strain is prescribed in a certain volume within an infinitely large homogeneous material. The prescribed strain is stressfree, i.e. no stress develops in as a result of this strain in an unconstrained setting. Accordingly this is referred to as an eigenstrain or a transformation strain. When constrained by the matrix, the equilibrium strain in the deformed region is given as:

$\tilde{\varepsilon}=\mathbb{E}: \varepsilon^{*}$,

where $\varepsilon^{*}$ is the eigenstrain, $\tilde{\varepsilon}$ is the equilibrium strain and $\mathbb{E}$ is the Eshelby tensor which is a function of the isotropic elastic properties of the matrix and the shape of the inclusion. For expressions of the Eshelby tensor for different geometries see for instance [13]. Here we use the one applicable to spherical inclusions.

Eshelby proposes that the stress concentrated in an inhomogeneity can be equally represented as the stress concentrated by an inclusion (occupying the same domain as the inhomogeneity) undergoing an arbitrary eigenstrain. In mathematical terms:

$\mathbb{C}_{I}:\left(\varepsilon_{M}+\tilde{\varepsilon}\right)=\mathbb{C}_{M}:\left(\varepsilon_{M}+\tilde{\varepsilon}-\varepsilon^{*}\right)$ 
where I and $\mathrm{M}$ denote the inhomogeneity and the matrix, respectively. Using Eq. 8 and considering that

$\boldsymbol{\varepsilon}_{I}=\boldsymbol{\varepsilon}_{M}+\tilde{\boldsymbol{\varepsilon}}$,

the strain concentration can be obtained in the following form:

$\boldsymbol{\varepsilon}_{I}=\left[\mathbb{E}:\left(\mathbb{C}_{M}^{-1}: \mathbb{C}_{I}-\mathbb{I}\right)+\mathbb{I}\right]^{-1}: \varepsilon_{M}$

This analytical solution is valid, as mentioned, for the case of a single inclusion and when the matrix is infinitely large. Therefore, it is not directly applicable in an RVE where the matrix is comparable in size to the inclusion. In order to use Eshelby's solution for homogenization, some assumptions have to be made. Therefore, following Eshelby's work, different homogenization schemes have been proposed which are based on different approaches to solve the problem.

\section{Self consistent method}

The Self Consistent scheme [8, 11] has been originally developed to compute the mechanical response of polycrystals and takes into account the interaction of the matrix and the grains using Eshelby's solution. In the model, every grain is treated as an inclusion in the overall RVE. Hence, based on Eq. 11 the strain concentration tensor for each grain becomes:

$\mathbb{A}_{k}=\left[\mathbb{E}:\left(\mathbb{C}^{-1}: \mathbb{C}_{k}-\mathbb{I}\right)+\mathbb{I}\right]^{-1}$

where $\mathbb{C}$ and $\mathbb{C}_{k}$ refer to the elasticity tensor of the RVE and the $k$ th grain, respectively. The Eshelby tensor in Eq. 12 is a function of the shape of the grain and the elastic properties of the RVE. The elasticity tensor of the RVE is computed as:

$\mathbb{C}=\sum_{k} f_{k} \mathbb{C}_{k}: \mathbb{A}_{k}$

Substituting Eqs. 13 in 12 makes it clear that this scheme has an implicit nature and requires a non-linear solution algorithm.

\section{Mori-Tanaka method}

For composite materials involving only two phases a simpler approach has been proposed by Tanaka and Mori [15]. In this scheme, it is assumed that the inclusions in the RVE, experience the matrix strain as the far-field strain in the Eshelby theory. This assumption allows:

$$
\begin{aligned}
\langle\boldsymbol{\varepsilon}\rangle_{\omega_{I}} & =\left[\mathbb{E}:\left(\mathbb{C}_{M}{ }^{-1}: \mathbb{C}_{I}-\mathbb{I}\right)+\mathbb{I}\right]^{-1}:\langle\boldsymbol{\varepsilon}\rangle_{\omega_{M}} \\
& \equiv \mathbb{H}:\langle\boldsymbol{\varepsilon}\rangle_{\omega_{M}},
\end{aligned}
$$

where $\mathbb{H}$ is a tensor that describes the relation between the strains in each phase. It is related to the strain concentration as:

$\mathbb{A}=\left[f \mathbb{I}+(1-f) \mathbb{H}^{-1}\right]^{-1}$.

The relation given in Eq. 14 approaches the exact solution as the volume fraction of the inclusions vanishes. Therefore, it can be deduced that the MoriTanaka assumption is well applicable for low secondphase concentrations.

For high volume fractions on the other hand Eq. 14 can be reversed to find:

$\langle\boldsymbol{\varepsilon}\rangle_{\omega_{I}}=\left[\mathbb{E}:\left(\mathbb{C}_{I}^{-1}: \mathbb{C}_{M}-\mathbb{I}\right)+\mathbb{I}\right]:\langle\boldsymbol{\varepsilon}\rangle_{\omega_{M}}$.

This property makes the Mori-Tanaka scheme attractive for two-phase composite materials with either low or high volume fraction of the inclusions. Furthermore, as seen in Eqs. 14 and 16 the scheme is explicit.

Lielens interpolation method

An alternative model for two-phase composites has been proposed by Lielens [2,3]. This model is based on an interpolation of the Mori-Tanaka scheme as a function of the volume fraction of the phases. With this approach the accuracy of the Mori-Tanaka scheme is increased for intermediate volume fractions. To calculate the strain concentration tensor in the Lielens algorithm the relation of the strains in each phase is formulated as:

$$
\begin{aligned}
\overrightarrow{\mathbb{H}} & =\left[\mathbb{E}:\left(\mathbb{C}_{M}^{-1}: \mathbb{C}_{I}-\mathbb{I}\right)+\mathbb{I}\right]^{-1}, \\
\overleftarrow{\mathbb{H}} & =\mathbb{E}:\left(\mathbb{C}_{I}^{-1}: \mathbb{C}_{M}-\mathbb{I}\right)+\mathbb{I}, \\
\langle\boldsymbol{\varepsilon}\rangle_{\omega_{I}} & =\left\{(1-\phi) \overrightarrow{\mathbb{H}}^{-1}+\phi \overleftarrow{\mathbb{H}}^{-1}\right\}^{-1}:\langle\boldsymbol{\varepsilon}\rangle_{\omega_{M}}
\end{aligned}
$$

where $\phi(f)$ is the chosen interpolation function. It can be observed that the tensors $\overrightarrow{\mathbb{H}}$ and $\overleftarrow{\mathbb{H}}$ relate to the forward and reverse Mori-Tanaka schemes, respectively. The requirement on $\phi(f)$ is that it should always be bracketed by 0 and 1 and approach to these values as $f$ approaches 0 and 1 , respectively. One function that meets these requirements is:

$\phi=f^{p}, \quad p \geq 0$,

where $p$ is introduced as a fitting parameter.

As seen in Eq. 17 the Lielens scheme is explicit and approaches the forward (actual) Mori-Tanaka for $f=0$ and the reverse Mori-Tanaka for $f=1$. The 
response of the composite for intermediate volume fractions is estimated by the interpolation of the two. However, a theoretical basis for that range is difficult to obtain since the terms "matrix" and "inclusion" become irrelevant. The Self Consistent scheme treats every phase as an inclusion in the composite material, but the validity of this approach is also questionable at these volume fractions. The introduced parameter $p$ in Eq. 17 becomes effective in this region and plays an important role on the stiffness in the intermediate ranges.

\section{Bound interpolation method}

Here, a simpler homogenization method for two-phase composites is proposed that does not require calculation of the Eshelby tensor. Instead, it relies on an interpolation function in order to describe the partitioning of the strain. The interpolation is based on the Voigt and Reuss models for changing volume fraction of the inclusions.

The strain relation between the phases is defined as:

$$
\langle\boldsymbol{\varepsilon}\rangle_{\omega_{I}}=\left[(1-\phi) \mathbb{C}_{M}^{-1}: \mathbb{C}_{I}+\phi \mathbb{I}\right]^{-1}:\langle\varepsilon\rangle_{\omega_{M}},
$$

where $\phi$ is the interpolation function. In the Lielens model (described above) the starting and end points are defined as the forward and the reverse Mori-Tanaka models, which are very accurate and accordingly the interpolation function starts at a value of 0 and ends at a value of 1 . In this scheme however the starting point is the Reuss assumption (lower bound) and the end point is the Voigt assumption (upper bound) which under and over estimate the stiffness, respectively. Hence, another interpolation function is proposed for $\phi$ as:

$\phi=\left(c_{1}+c_{2} f\right)^{c_{3}}$

where $c_{1-3}$ are fitting parameters. In order not to violate the theoretical requirements, the parameters chosen must satisfy the following conditions:

$0 \leq c_{1}^{c_{3}} \leq 1$,

$0 \leq\left(c_{1}+c_{2}\right)^{c_{3}} \leq 1$.

The proposed technique can alternatively be derived from the Mori-Tanaka scheme. Starting from the strain concentration tensor in Eq. 14, if the Eshelby tensor $\mathbb{E}$ is replaced with the spherical tensor:

$\mathbb{E}=(1-\phi) \mathbb{I}$,

Equation 19 is obtained. This derivation also shows that in the cases where the Eshelby tensor is anisotropic, e.g. the inhomogeneity is not spherical in shape, the anisotropy expected from the material cannot be captured with this approach.

\section{Elastic-plastic homogenization}

The equations presented in the previous section are based on the elastic properties of the constituents. In order to utilize them in an elastic-plastic homogenization framework a fictitious reference material must be chosen. Hill [7] introduced as reference the linearized elastic-plastic response of the phases, i.e. the continuum elastic-plastic modulus. With this approach the stiffness of each phase is approximated by its instantaneous modulus which is a function of deformation. These moduli are then used for determining the strain concentration tensors of the phases. Alternative definitions of the reference material also exist in literature. In Moulinec and Suquet [12], the advantages of using the secant modulus over the elastic-plastic one is discussed. It is shown on a number of problems that algorithms using the secant modulus compare better with experiments. In Doghri and Ouaar [3], the advantages of using the continuum tangent modulus and the algorithmic tangent modulus are compared, including their isotropic projections. It is demonstrated that using the algorithmic tangent moduli as reference materials and the isotropic projection of them in computing the Eshelby tensor results in good agreement with observations.

From these studies it is clear that the selection of the reference material is very important to have a reliable algorithm. In this study the algorithmic elastic-plastic tangent modulus is chosen as the reference material. For calculating the Eshelby tensor, the isotropic projection of the corresponding tangent is used (for details of the projection algorithm see [3]) and the inclusions are considered to be spherical in shape.

In large deformation analysis, as mentioned before, care must be taken in relying on the averaging operator. When the deformed configuration does not coincide with the reference configuration, some of the averaged strain and stress measures do not conform with their macroscopic values. Nemat and Nasser [14] gives a detailed analysis on this matter. For the case of forming operations, an approximation to the solution can be obtained using an incremental approach where the total deformation is imposed incrementally on the RVE and the configuration is updated after each converged step. Provided that the incremental displacements are small compared to the size of the RVE, the current configuration can be approximated with the reference configuration. This implies that the effects of the inconsistencies are negligible. 
According to these considerations, in the end a symmetric strain concentration tensor is obtained. Therefore, when the velocity gradient $(\mathbf{L}=\nabla \mathbf{v})$ is used as a strain measure, only the symmetric part of it $(\mathbf{D}=$ $\left.\frac{1}{2}\left(\mathbf{L}+\mathbf{L}^{T}\right)\right)$, i.e. deformation rate, will be concentrated and anti-symmetric part $\left(\mathbf{W}=\frac{1}{2}\left(\mathbf{L}-\mathbf{L}^{T}\right)\right)$, i.e. spin, will be unaffected. Assuming rotational equivalence of the phases results in the following formulation:

$$
\begin{aligned}
& \langle\mathbf{D}\rangle_{\omega_{k}}=\mathbb{A}_{k}:\langle\mathbf{D}\rangle_{\omega}, \\
& \langle\mathbf{W}\rangle_{\omega_{k}}=\langle\mathbf{W}\rangle_{\omega} .
\end{aligned}
$$

Equation 22 implies that the strain concentration tensor operates on the deformation rate of each phase and the rotation at the macroscale is considered as a rigid body rotation over the entire RVE.

\section{Solution algorithms}

For the elastic homogenization, i.e. when both phases remain elastic during the deformation, the solution of the strain partitioning according to the schemes described in the previous section is trivial (except for the Self Consistent scheme which will be treated below).

For the elastic-plastic case, the strain concentration tensor becomes a function of the deformation. This means that although the schemes Voigt, Reuss, MoriTanaka, Lielens Interpolation and Bound Interpolation yield an explicit definition of the concentration tensor, its computation requires an iterative algorithm. In Doghri and Ouaar [3], the solution is obtained using the method of Fixed-Point iterations which proves to be robust for this problem.

The non-linearity arises due to the fact that the strain concentration tensor for all the schemes is a function of the instantaneous moduli of the phases. As these moduli change with the amount of strain increment so does the partitioning. In an Euler backward (fully implicit) approach therefore the system of equations becomes non-linear. In order to solve the system, the stress update is considered to take place in two levels where the homogenization level encapsulates the stress-update of each phase.

The algorithm starts with an initial partitioning of the phases according to the Voigt assumption. Having the initial strain increment for each phase, the stresses are updated and the corresponding moduli of the phases are determined. Based on these the strain concentration tensor is determined which results in the new strain increments. The iterations continue until there is no change in the concentrated strain. It is worth mentioning that the homogenization algorithm is independent of the constitutive models chosen for each phase. The algorithm is summarized in Algorithm 1.

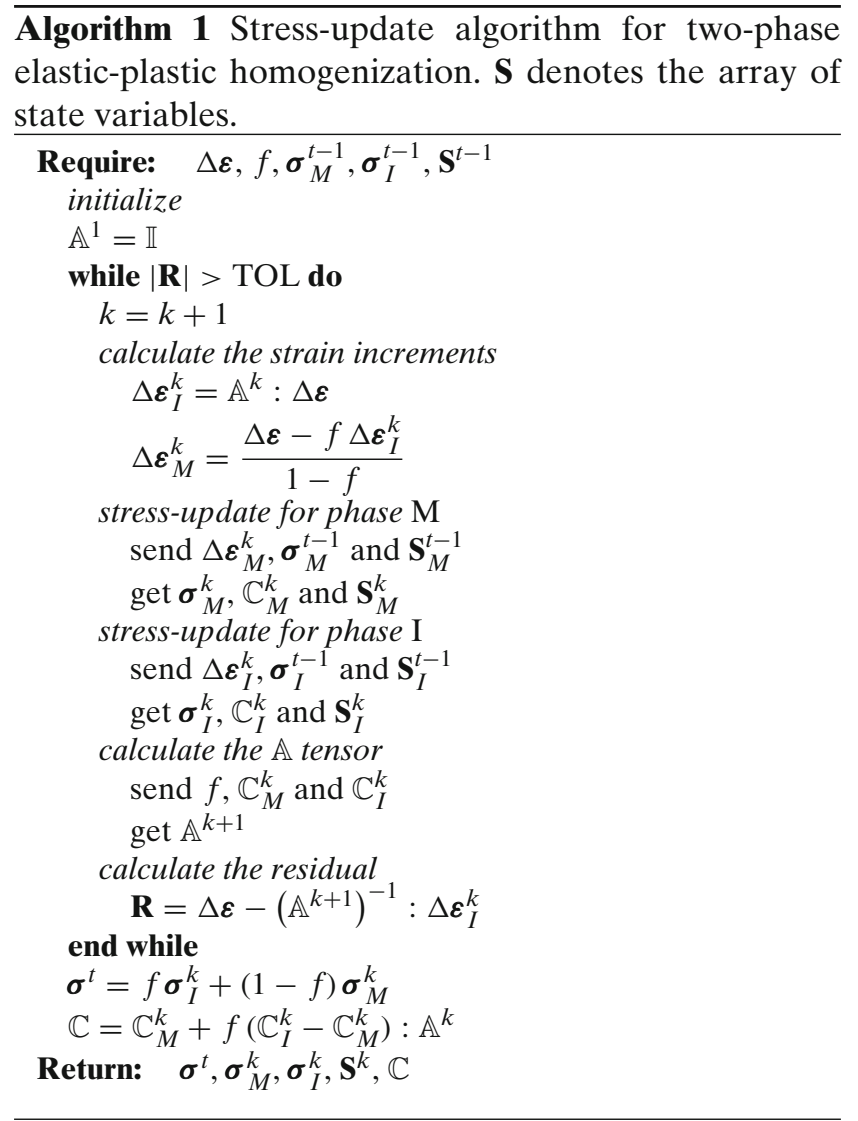

The calculation of the $\mathbb{A}$ tensor is trivial for all schemes except for the Self Consistent due to its implicit nature. The iterative algorithm for the solution of the strain concentration for this case is given in Algorithm 2.

Plane stress condition

An important constraint for sheet-metal forming simulations is the plane stress condition which stems from the fact that the thickness of the sheet metal is very small compared to the in-plane dimensions. This condition requires the stress components in the thickness direction to vanish. Accordingly, the thickness strain is an unknown that has to be determined.

Although for a homogeneous material the strain can be assumed to be uniform over a material point (considering that the material point comprises sufficiently many grains), for a two-phase composite material a uniform thickness strain assumption does not hold. In case the plane stress condition is imposed on each phase individually the system will be over-constrained. The extra constraint makes the solution depart from the minimum energy solution. Therefore it must be kept 


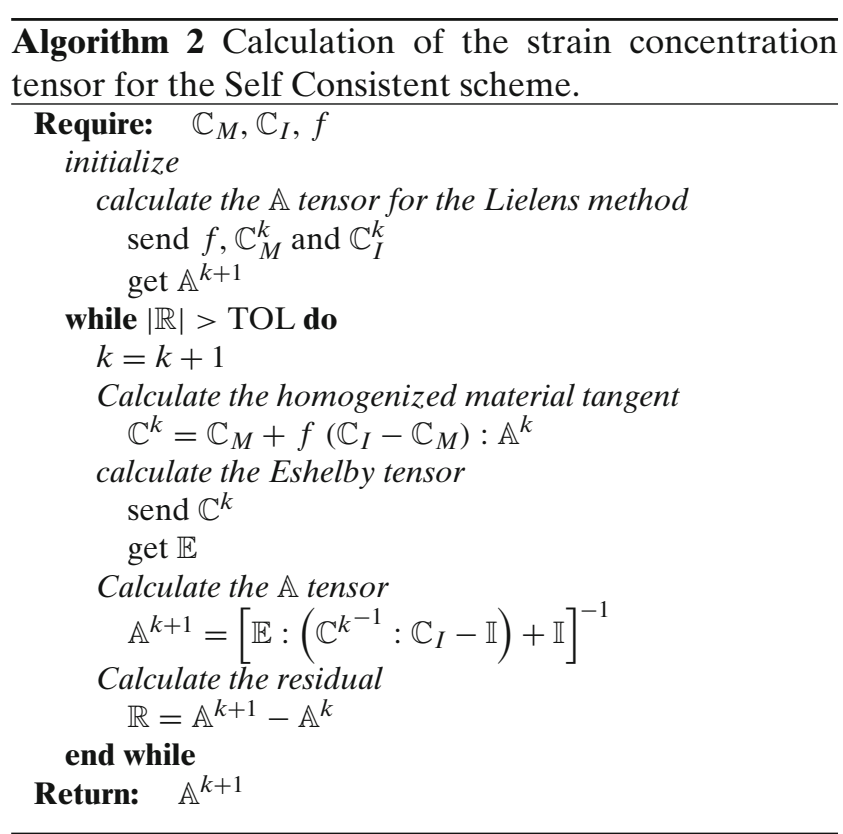

in mind that when there are constraints over the RVE it is crucial to impose these conditions on the overall behavior of the RVE and not on the individual phases.

This however, leads to a computational drawback. Since the plane stress condition cannot be imposed on each phase, the stresses must be computed for the full three dimensional case. Additionally, the resulting material tangent must be condensed.

In this study the Regula-Falsi (False Position) method is used to solve for the thickness strain. The condensation of the material tangent for the plane stress condition is performed as:

$$
\begin{aligned}
& {\left[\begin{array}{c}
\dot{\boldsymbol{\sigma}}_{a} \\
\mathbf{0}
\end{array}\right]=\left[\begin{array}{ll}
\mathbb{C}_{a a} & \mathbb{C}_{a b} \\
\mathbb{C}_{b a} & \mathbb{C}_{b b}
\end{array}\right]\left[\begin{array}{c}
\dot{\boldsymbol{\varepsilon}}_{a} \\
\dot{\boldsymbol{\varepsilon}}_{b}
\end{array}\right]} \\
& \Rightarrow \dot{\boldsymbol{\sigma}}_{a}=\left(\mathbb{C}_{a a}-\mathbb{C}_{a b}: \mathbb{C}_{b b}^{-1}: \mathbb{C}_{b a}\right): \dot{\boldsymbol{\varepsilon}}_{a}
\end{aligned}
$$

where $a$ and $b$ stand for the in-plane and out-of-plane components, respectively.

\section{Results}

The results of the constitutive model are presented first at the material point level where the schemes are compared with each other. Next, as a demonstration of the model, a deep drawing simulation run in MSC.Marc is shown.

For the demonstrations, a simple J2-plasticity model with isotropic hardening is assumed for each phase. Hardening is prescribed as a power-law relation. However, the homogenization method presented in this arti-
Table 1 Plasticity material paramaters

\begin{tabular}{lccl}
\hline Phase & $\sigma^{y}(\mathrm{MPa})$ & $K(\mathrm{MPa})$ & $m$ \\
\hline Ferrite $(\alpha)$ & 291 & 741 & 0.1928 \\
Martensite $\left(\alpha^{\prime}\right)$ & 1,293 & 2,486 & 0.0747 \\
\hline
\end{tabular}

cle is not restricted by the material model chosen for the constituent phases. The material parameters are chosen for a DP600 dual-phase steel, where the constituent phases are ferrite $(\alpha)$ and martensite $\left(\alpha^{\prime}\right)$. The phase fraction of martensite is considered as: $f=0.2$.

Elastic properties of both phases are assumed to be equal, which is a reasonable assumption for DP steels. The elastic modulus and the Poisson's ratio are chosen as: $E=210(\mathrm{GPa})$ and $v=0.3$. The plastic material parameters are summarized in Table 1 which describe the flow stress in the form: $\sigma^{f}=\sigma^{y}+K\left(\varepsilon^{p}\right)^{m}$.

Partition of strain and stress

In this section all the discussed homogenization schemes are demonstrated on a test problem at the integration point level. The aim is to show how the strain and stress are partitioned into the phases for a prescribed stress in the overall material. For the solution of the strain increments, the material tangent computed by the homogenization algorithm is used. The tangent proves to supply stable convergence behavior however due to the history-dependent nature small increments lead to more accurate results.

In the example, a uniaxial stress state is prescribed and the stresses and strains in the phases are computed with the homogenization schemes described in the previous section. The only schemes that need input parameters are Lielens interpolation and Bound interpolation, of which the former has one and the latter has three. Here, the parameters are selected such that the results closely approximate the Self Consistent scheme. On the other hand the advantage of these models is that if data from an experiment exists, the response can be adjusted accordingly. The chosen parameters are:

$p=0.75$,

$c_{1}=0.6, c_{2}=0.4, c_{3}=1.2$.

In Fig. 1 the equivalent stress results obtained using all the schemes are presented. As expected Voigt and Reuss models give the most stiff and most compliant response, respectively. Among the schemes, Self Consistent and Mori-Tanaka models do not have any fitting parameters and the results show that the latter has softer response than the former. The results of the proposed Lielens interpolation and Bound Interpola- 


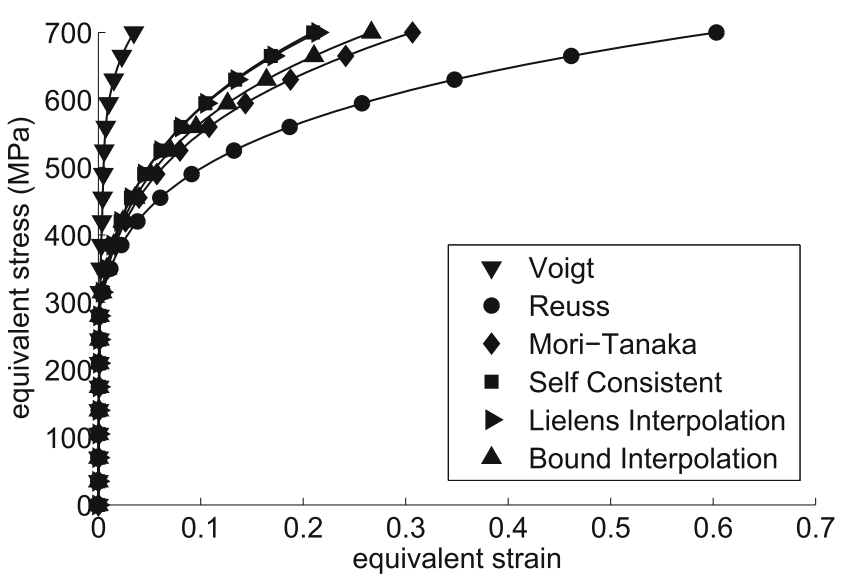

Fig. 1 Comparison of the stress-strain relations obtained with different homogenization schemes under uniaxial tension

tion schemes lie in between Self Consistent and MoriTanaka models. This is due to the chosen parameters for the interpolation. With different parameters the response can be altered to be stiffer or more compliant.

Figures 2 and 3 show the developed normal stresses in the orthogonal direction in each phase. As discussed before these stress components are not zero, as opposed to the overall stress. This means that although the averaged response of the material is uniaxial, in the individual phase level it is not. If this condition would have been imposed on each phase, the results would deviate from the current ones.

It can be seen on the results that all schemes, except for the bounds, capture similar directions of the stresses that develop in each phase. Although the Bound Interpolation model is not based on the Eshelby's solution, it is observed that the direction predicted by it matches those by the Self Consistent and the Lielens Interpolation models. On the other hand, this observation is

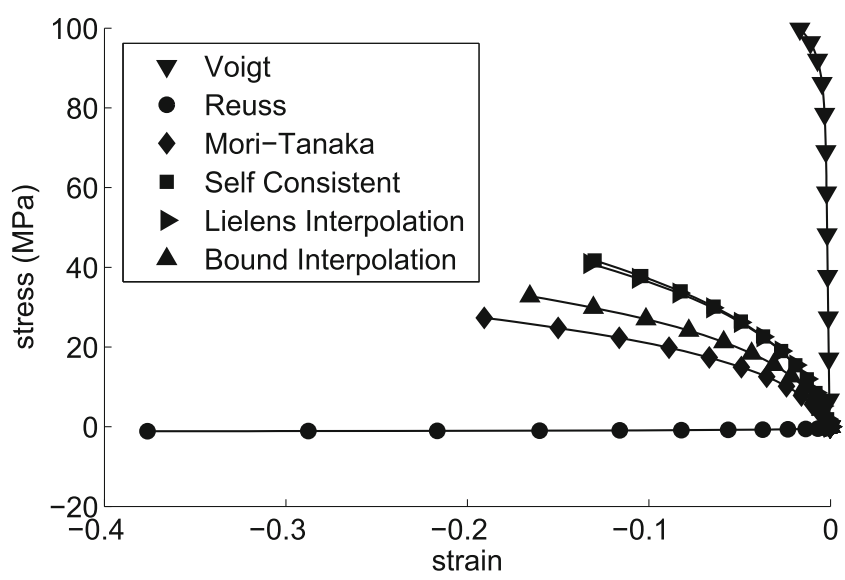

Fig. 2 Comparison of the austenite normal stress in the orthogonal direction for different homogenization schemes

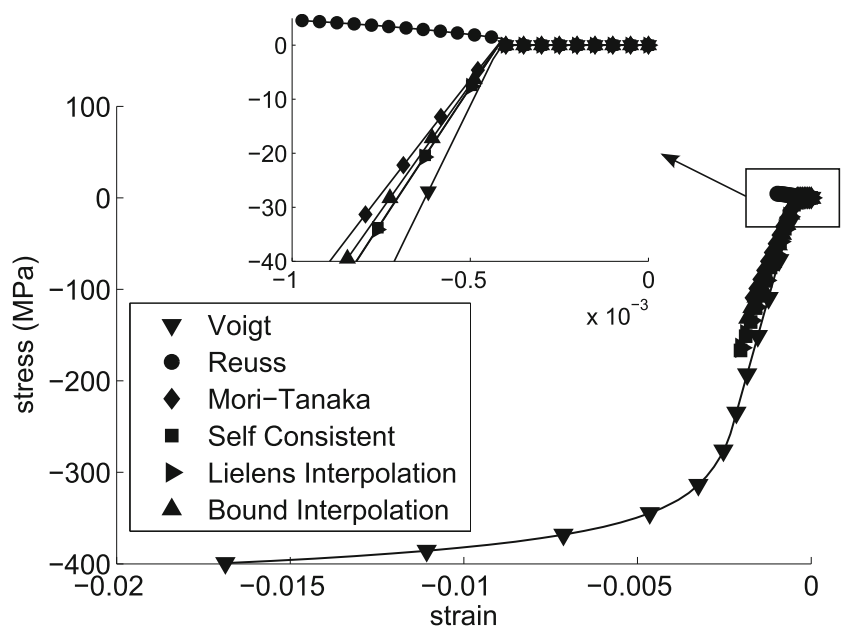

Fig. 3 Comparison of the martensite normal stress in the orthogonal direction for different homogenization schemes

true only for isotropic material properties and spherical inclusions.

\section{Cyclic shear}

In this section the results of the different schemes for a plane-stress, cyclic shear deformation are presented. The constitutive models of each phase involve only isotropic hardening. This means that the phases individually cannot capture the Bauschinger effect. In order to have that possibility a kinematic hardening model has to be included.

On the other hand, in the two phase composite due to differences in the yield stresses of the phases, the Bauschinger effect can be observed clearly. This arises due to the fact that during reverse loading when the soft phase already starts developing a negative stress, the hard phase is still elastic with a positive stress. As the soft phase yields in the reverse direction-at its isotropic flow stress - the average stress over the composite is smaller in magnitude than the forward flow stress. The additional influence of kinematic hardening models for the constituent phases have been studied in Doghri and Friebel [2] by cyclic deformation simulations for the Mori-Tanaka and Lielens Interpolation schemes.

The results of the schemes in cyclic shear are shown in Fig. 4. It is seen that all models except Reuss show the Bauschinger effect. It is most pronounced in the Voigt model while the Self Consistent and Lielens Interpolation schemes also capture the effect significantly. On the other hand, after the second direction change it is seen that all the models return to their original flow stress relation. 


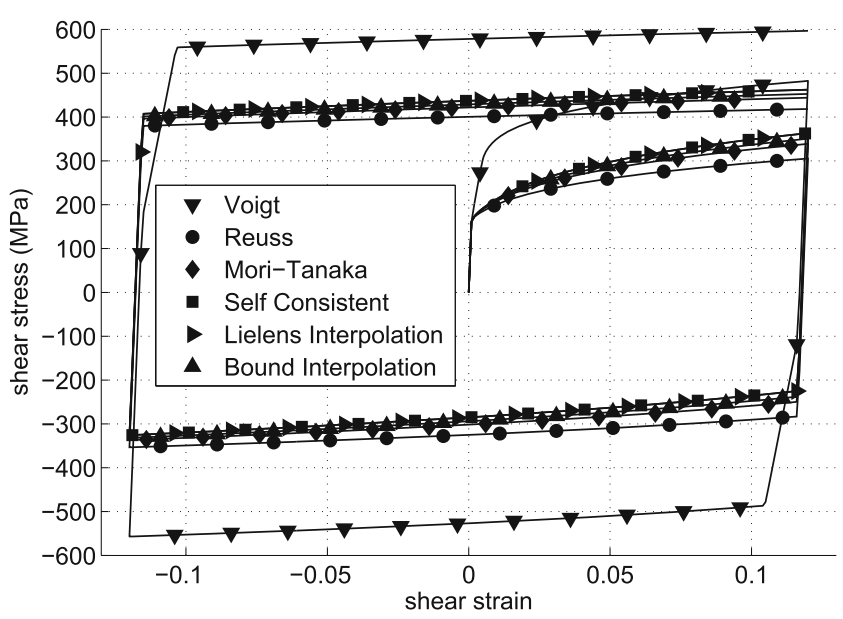

Fig. 4 Comparison of the shear stress in a cyclic test for different homogenization schemes

\section{Sheet metal forming simulation}

Here, the applicability of the presented schemes in a full scale FE simulation is presented. The process is cup-drawing of a thin sheet with the parameters given as:

$\begin{array}{rrr}\text { Blank radius: } & 90 & \mathrm{~mm} \\ \text { Blank thickness: } & 1 & \mathrm{~mm} \\ \text { Punch radius: } & 50 & \mathrm{~mm} \\ \text { Die radius: } & 51.25 & \mathrm{~mm} \\ \text { Punch fillet radius: } & 9.53 & \mathrm{~mm} \\ \text { Die fillet radius: } & 7.14 & \mathrm{~mm} \\ \text { Friction coefficient: } & 0.05 & \end{array}$

In the simulation a quarter of the blank is discretized with 360 thick shell elements which have out-of-plane shear components and reduced integration capability.

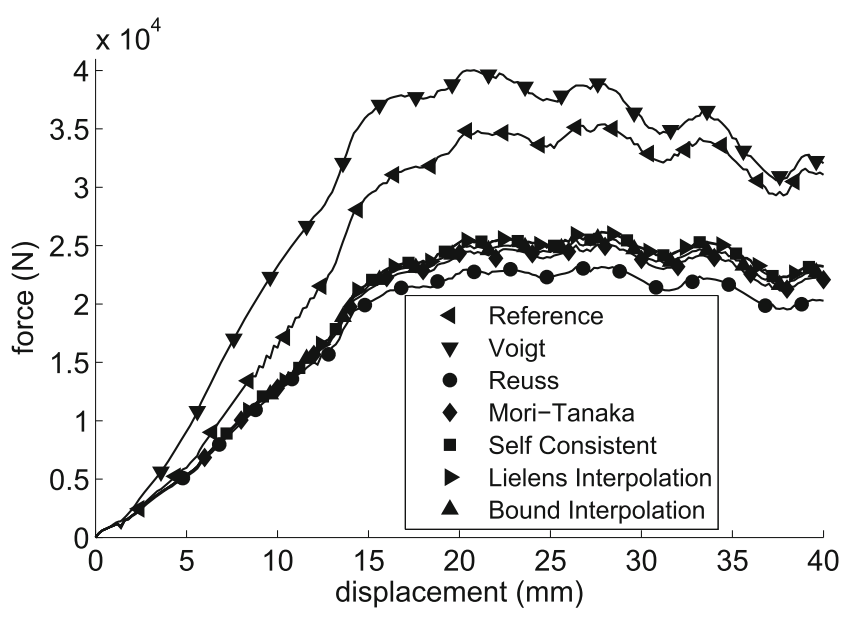

Fig. 5 Punch force-displacement curves obtained using different homogenization schemes

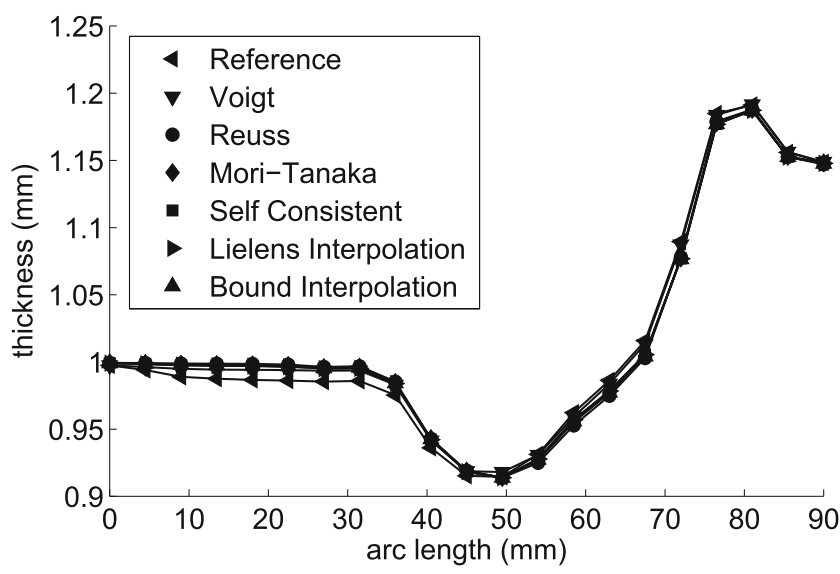

Fig. 6 Thickness profiles obtained using different homogenization schemes

The punch force-displacement curves obtained using the homogenization schemes are presented in Fig. 5. As a reference, results from a single phase, isotropic material model that is fitted to the Self Consistent uniaxial hardening curve is also shown. In the figure it is observed that the results of the homogenization schemes show the similar trend as in the integration point level results. The single phase curve however falls significantly apart. This is related to the fact that the partitioning of stress into phases depends on the current stress state as shown on the previous examples. Additionally, homogenization schemes show the Bauschinger effect whereas the single phase model involves only isotropic hardening.

In Fig. 6, the thickness distribution over the blank radius is plotted. No significant difference is observed among the different models.

In order to compare the computational efficiency of the models the CPU times as well as the global number of iterations are summarized in Table 2. No significant difference in the number of iterations can be observed among the models. In the computational costs however the Self Consistent scheme stands out as the

Table 2 CPU times and global number of iterations required for the simulation using different homogenization schemes

\begin{tabular}{lcl}
\hline Scheme & CPU time (s) & Iterations \\
\hline Reference & 97 & 618 \\
Voigt & 569 & 602 \\
Reuss & 620 & 608 \\
Mori-Tanaka & 652 & 615 \\
Self consistent & 2,133 & 638 \\
Lielens interpolation & 814 & 641 \\
Bound interpolation & 640 & 643 \\
\hline
\end{tabular}


most demanding one. Lielens and Bound interpolation schemes therefore serve as cheap alternatives which are shown to yield accurate results.

\section{Summary and conclusions}

The study concerns the Mean-Field homogenization method to be applied in constitutive modeling of multiphase materials. The goal of this study was to use a physically based approach and develop computationally efficient algorithms which can be used in modeling of these materials.

The basic ingredients of the Mean-Field homogenization method as well as the details of different homogenization schemes were presented. The Voigt and Reuss schemes are based on very crude assumptions and determine the upper and the lower bounds for the incremental stiffness of the material. Two completely physically based schemes can be found in literature, namely the Self Consistent and Mori-Tanaka approaches. These schemes do not involve any fitting parameters and capture the response of the composite based on Eshelby's solution of the inhomogeneity problem. The Mori-Tanaka scheme is accurate for low volume fractions of the inclusions whereas there is no such restriction on the Self Consistent model.

The Lielens scheme interpolates the forward and reverse Mori-Tanaka models to capture the response at higher concentration of the inclusions. A new interpolation function is proposed here with which a fitting parameter is introduced. The parameter does not affect the results at low and high volume fractions but enables to alter the behavior in the intermediate range.

A new scheme is proposed which is similarly an interpolative method. The schemes that are interpolated in this case are Voigt and Reuss. This method is not based on the Eshelby's solution and is rather heuristic. However a derivation based on the Mori-Tanaka approach justifies the use of the model. The interpolation function has three parameters which makes the fitting of the response flexible.

The schemes are demonstrated at the integration point level on one stress and one strain driven example. The results reveal that all schemes, except for the bounds, yield similar results. An important consideration here is the direction of stresses that develop within each phase. It is seen that the proposed schemes can capture the directions accurately.

Finally, the models are compared on a full scale deep-drawing simulation. It is observed that the con- vergence behavior of homogenization schemes is comparable with conventional plasticity models. In terms of computational efficiency the schemes perform satisfactorily where only less than an order of magnitude of increase in time is observed.

Acknowledgement This research was carried out under project number M63.1.09373 in the framework of the Research Program of the Materials innovation institute M2i (www.m2i.nl).

Open Access This article is distributed under the terms of the Creative Commons Attribution Noncommercial License which permits any noncommercial use, distribution, and reproduction in any medium, provided the original author(s) and source are credited.

\section{References}

1. Doghri I (2000) Mechanics of deformable solids. Springer

2. Doghri I, Friebel C (2005) Effective elasto-plastic properties of inclusion-reinforced composites. study of shape, orientation and cyclic response. Mech Mater 37:45-68

3. Doghri I, Ouaar A (2003) Homogenization of two-phase elasto-plastic composite materials and structures study of tangent operators, cyclic plasticity and numerical algorithms. Int J Solids Struct 40:1681-1712

4. Eshelby J (1957) The determination of the elastic field of an ellipsoidal inclusion, and related problems. Proc R Soc Lond A 241(1226):376-396

5. Gonzáles C, Llorca J (2000) A self-consistent approach to the elasto-plastic behaviour of two-phase materials including damage. J Mech Phys Solids 48:675-692

6. Hill R (1963) Elastic properties of reinforced solids: some theoretical principles. J Mech Phys Solids 11:357-372

7. Hill R (1965a) Continuum micro-mechanics of elastoplastic polycrystals. J Mech Phys Solids 13:89-101

8. Hill R (1965b) A self-consistent mechanics of composite materials. J Mech Phys Solids 13:213-222

9. Kouznetsova V, Brekelmans W, Baaijens F (2001) An approach to micro-macro modeling of heterogeneous materials. Comput Mech 27:37-48

10. Kouznetsova V, Geers M, Brekelmans W (2004) Multiscale second-order homogenization of multi-phase materials: a nested finite element solution strategy. Comput Methods Appl Mech Eng 193:5525-5550

11. Kröner E (1958) Berechnung der elastischen Konstanten des Vielkristalls aus den Konstanten des Einkristalls Z. Phys 151:504-518

12. Moulinec H, Suquet $P$ (2003) Intraphase strain heterogeneity in nonlinear composites: a computational approach. Eur J Mech A Solids 22:751-770

13. Mura T (1982) Micromechanics of defects in solids. Martinus Nijhoff Publishers

14. Nemat-Nasser S (1999) Averaging theorems in finite deformation plasticity. Mech Mater 31:493-523

15. Tanaka K, Mori T (1970) The hardening of crystals by nondeforming particles and fibres. Acta Metall 18:931-941 